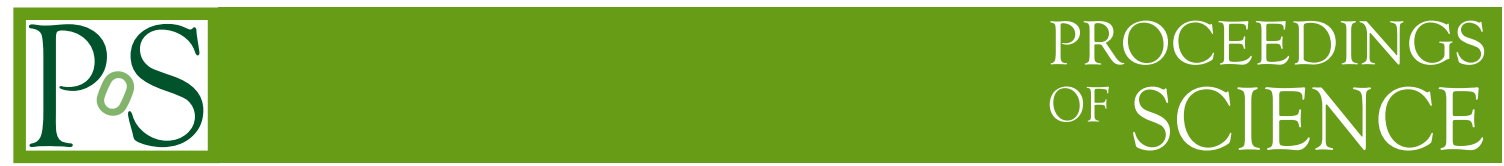

\title{
Dark Cosmological Simulations
}

\author{
Raúl E. Angulo* \\ Centro de Estudios de Física del Cosmos de Aragón (CEFCA), Teruel, Spain. \\ E-mail: rangulo@cefca.es
}

\begin{abstract}
Numerical simulations have a prime role in modern cosmology. Here I review the state-of-the-art in this field discussing the importance of dark matter N-body simulations in our understanding of the dark universe. I will put their use in the context of the latest generation of wide-field galaxy surveys and dark matter searches. I also address the shortcomings of current approaches, and discuss recent developments in solving the N-body problem. I finish with an outlook for possible developments foreseen in the near future.
\end{abstract}

Frank N. Bash Symposium 2013: New Horizons in Astronomy (BASH 2013)

October 6-8, 2013

Austin, Texas

${ }^{*}$ Speaker. 


\section{Introduction}

Our current understanding of structure formation in the Universe stands on four pillars: I) the existence of Dark Energy (DE) in the form of a cosmological constant $(\Lambda)$; ii) Cold Dark Matter $(\mathrm{CDM})$, as the main gravitating component in the cosmos; iii) Gaussian primordial density fluctuations in the early Universe; and iv) General Relativity as the law of gravity. All these ingredients together form the so-called Lambda Cold Dark Matter $(\Lambda \mathrm{CDM})$ cosmological model. This model has been supported over the last 20 years by many different astrophysical observations, ranging from the anisotropies in the cosmic microwave background radiation, over weak and strong gravitational lensing, to the topology of the large-scale distribution of galaxies, among many other observations (e.g., $[17,19,12])$. In general, $\Lambda \mathrm{CDM}$ is the simplest explanation for the accelerated expansion of the Universe and the wealth of structure we see within.

Numerical simulations have been essential in the establishment of this "standard model" in cosmology: the first indication that the expansion of the Universe is accelerating came from comparing cosmological simulations with observations of the distribution of galaxies on the sky [6]. The cosmic acceleration was measured a few years later using Type Ia Supernovae. Numerical simulations are also invaluable cosmological tools: They are the most reliable and precise method to follow the highly nonlinear evolution of primordial density fluctuations (e.g., [14]). Hence, they provide virtual universes with which we can test the predictions of $\Lambda \mathrm{CDM}$ and interpret astronomical observations.

Contrasting the successes of $\Lambda \mathrm{CDM}$ in predicting the properties of the cosmic structures we observe, there are fundamental open questions about each of its pillars. General relativity has only been accurately tested on scales much smaller than those relevant for cosmology. Dark matter might be self-interacting and/or an elementary particle that is not completely cold, but warm. And almost every theory that explains either the accelerated expansion of the Universe or Inflation also predicts a Dark Energy equation of state varying with time or (slightly) non-Gaussian primordial density fluctuations, respectively.

These enigmas have driven the design and construction multi-million dollar experiments. The largest galaxy surveys ever carried out have started scanning the sky with unprecedented detail, providing extremely precise measurements of the large-scale galaxy distribution and gravitational lensing signal. Similarly, many experiments are specially designed to directly or indirectly interact with the dark matter particles, placing constraints in the mass and cross section of this hypothetical new particle. All this could help us solving the mysteries of $\Lambda \mathrm{CDM}$, which could even lead to the discovery of completely new physical laws.

The signatures of departures from $\Lambda \mathrm{CDM}$ depend on the detailed distribution of dark matter, on the precise impact of dark energy on cosmic structure, and on the nonlinear tracers, from gigaparsecs down to subgalactic scales. Therefore, the interpretation of future precision measurements of, for instance, the structure in the Ly- $\alpha$ forest, in the galaxy and galaxy cluster distributions, from weak gravitational lensing, or searching for primordial nongaussianity, will rely heavily on N-body simulations since they provide the only accurate account of nonlinear evolution from the initial conditions provided by early universe physics. Thus, modern cosmological simulations face new challenges in terms of their accuracy and predictive power.

In this document I will describe the state-of-the-art cosmological $N$-body simulations, empha- 
sizing their connection with cosmological experiments. I will also highlight new developments in the algorithms employed, and efforts in simulating exotic cosmological components. I will conclude with directions for future progress.

\section{Simulating the Dynamics of Cold Dark Matter}

In $\Lambda \mathrm{CDM}$, most of the mass in the Universe is in the form of an unknown elementary particle: the Dark Matter (DM). Observations indicate that this particle is in a "cold" state (i.e. has small thermal velocity) and interacts mainly gravitationally. Also, after the last scattering surface, there were only very tiny fluctuations in Dark Matter density, but that extended down to very small scales (possibly down to an Earth mass!). These peculiar properties of the DM particle make it hard to detect observationally, but they make the study of the formation of structures in the Universe a well-posed and ideal problem for cosmological collisionless N-body simulations.

The gravitational interaction of a large number of micro-physical DM particles can be described by the the Collisionless Boltzman equation (also known as Vlassov-Poisson), which solves for the evolution of the full phase-space distribution function, $f=f(\mathbf{x}, \mathbf{v})$ (the number of particles in a phase-space volume), and reads:

$$
0=\frac{\mathrm{d} f}{\mathrm{~d} t}=\frac{\partial f}{\partial t}+\frac{\mathbf{v}}{a^{2}} \cdot \frac{\partial f}{\partial \mathbf{v}}-\frac{\partial f}{\partial \mathbf{x}} \frac{\partial \Phi}{\partial \mathbf{x}},
$$

where $\Phi$ is the gravitational potential, which is related to the mass distribution via the Poisson equation:

$$
\nabla^{2} \Phi=\frac{4 \pi G}{a} \int f \mathrm{~d}^{3} v
$$

where $G$ is the gravitational constant and $a$ is the scale factor given by the Friedmann equation. The above equations are in general six-dimensional, however, for cold dark matter the distribution function occupies only a 3-D surface of the 6-D phase space. Even with this simplification, directly solving these equations is in practice prohibitively expensive computationally. Although there are some explicit solutions for a restricted set of cases, it is common to assume that the evolution of $f$ is analogous to the that of a coarse-grained distribution function (that represents an average of the microscopic position and velocity distribution functions of the full CDM fluid).

\subsection{Standard Approaches}

The $N$-body method solves for the evolution of the coarse-grained distribution function in a MonteCarlo fashion. A set of discrete simulation bodies, or "particles", represents the full mass distribution, and their dynamic is governed by:

$$
\begin{aligned}
\frac{\mathrm{d}^{2} \mathbf{x}_{i}}{\mathrm{~d}^{2} t} & =\nabla_{i} \Phi\left(\mathbf{x}_{i}\right) \\
\Phi(\mathbf{x}) & =-G \sum_{i} \frac{m_{i}}{\left[\left(\mathbf{x}_{i}-\mathbf{x}\right)^{2}+\varepsilon^{2}\right]}
\end{aligned}
$$


where the subscript $i$ runs over all simulation particles, and $m_{i}$ is the mass of the respective simulation particle. The parameter $\varepsilon$ is usually referred to as "softening length" and prevents forces from diverging, which in turn suppresses numerical artefacts such as 2-body wide-angle scatterings.

It is well known in gravitational dynamics that a discrete system will not behave in detail in the same manner as another that represents the same mass distribution but with many more simulation particles (e.g., the importance of phase and chaotic mixing, violent relaxation, Landau damping will be different in the two systems). However, most simulation results do not appear to be seriously affected by this: their predictions display very well-behaved convergence properties when $N$ is varied. This fact supports the coarse-fined grained correspondence.

\subsection{New approaches}

A different way of carrying out the $N$-body method, and thus for simulating the DM evolution, has been proposed recently $[1,20,9]$. Instead of assuming that simulation particles carry mass, the new method assumes that they are massless and that they simply trace the gravitational distortion of initial coarse-grained phase-space volume elements. The DM mass to be simulated is uniformly distributed inside these volume elements. At any time in the simulation, the mass field (and the corresponding solution of the Poisson equation) is given by integrating over all overlapping phasespace volume elements.

This simple new idea results in a continuous and smooth density and force fields, which suppresses drastically the effect of particle discreteness in $\mathrm{N}$-body simulations (it also removes the need of a softening length). To illustrate the advantage of the new scheme, Fig. 1 shows a comparison of the projected density field in the outskirts of a DM halo, as computed by a traditional $N$-body method (right panel) and by the new method (left panel). The improvement in the description of the cosmic field is evident - halos, filaments and caustics are much better resolved. This directly translates into a different evolution of the DM fluid that outperforms standard approaches very clearly in determined cases, such as those where the primordial density fluctuations power spectrum have a small-scale cut-off [3].

Unfortunately, density fields calculated in this way are biased if the distortion of an initial phase-space volume cannot be represented by linear transformations. In cosmological simulations this happens in two situations. i) In collapsed structures, which have at their centers high densities and short dynamical time-scales. There, densities are systematically overestimated at the halo center and slightly underestimated in outer regions. The second situation is in halo substructures, where phase-volume elements can be significantly stretched when mass is stripped out by tidal forces. There, the net effect is an underestimation of the mass associated with substructures.

These limitations are likely to be solved by further developments of the method. In particular, several directions are being actively pursued regarding a dynamic and adaptive refinement of the phase-space volume elements. This will allow following more accurately (and for longer) the highly nonlinear DM evolution, increasing the resolution in places where it is required. If these developments alleviate the problems outlined above, this new approach has the potential to completely replace the standard one in the future. 


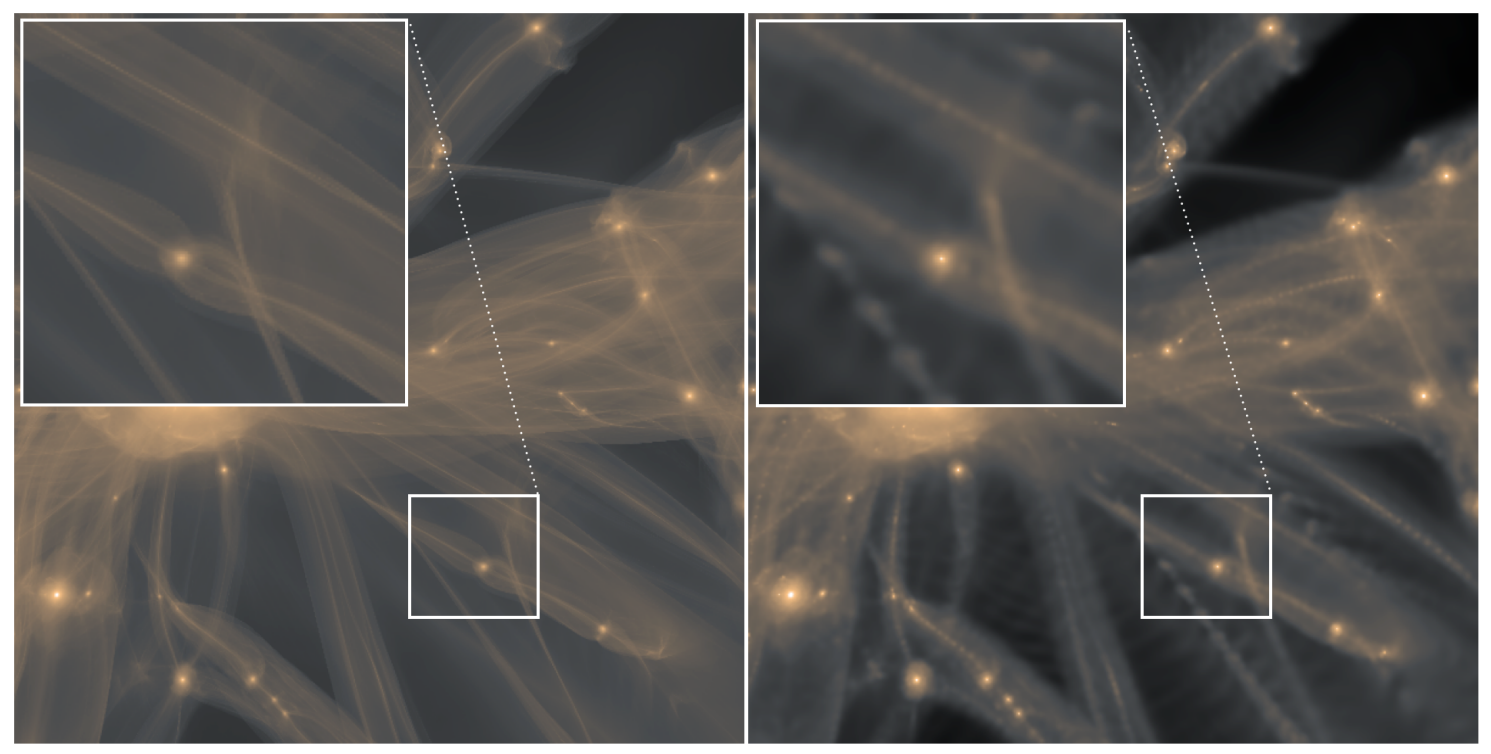

Figure 1: A comparison between the projected density field using a standard $N$-body approach employing an adaptive kernel smoothing (right panel) and a recently proposed new scheme (left). Artefacts due to the poor density estimates in low-density regions are obvious in the traditional method, whereas the new approach achieves a representation of small and large structures with much less noise. Adopted from [11]

\section{State of the Art}

The set of equations solved by dark-matter $N$-body simulations represents well-known physical laws (as opposed to hydrodynamical simulations, where many of the physical processes involved are largely unconstrained at the relevant scales). In addition, the algorithms employed in their solution have reached maturity over many years of development. Because of these, the main limitation of dark cosmological simulations is in efficiency of the implementation of the algorithms, in particular to their parallelization, and in the available computational power.

The processing capabilities of supercomputers have been steadily increasing over the last 40 years in an exponential way - roughly doubling the number of transistors in CPUs (which is known as Moore's law). Cosmological simulations exploited this, roughly doubling in particle number every 17 months since the 1970s, and constantly improving their accuracy and precision. Simulations have provided insights into regimes inaccessible by analytical techniques, which have resulted into an enormous contribution to our understanding of the Universe. In the future, hardware capabilities are foreseen to keep increasing (albeit with more complex architectures), with the exaflop limit expected to be reached by 2020. It is not hard to anticipate that dark simulations will continue to systematically push the envelope of what is feasible at international supercomputer centres.

Areas where simulations of representative regions of the cosmos have helped is in the design and exploitation of large galaxy surveys. On the other hand, simulations focusing on individual collapsed DM structures ("halos") have provided predictions for the distribution of DM around us, guiding experimental searches for i) collisions of DM particles in detectors on earth, and of ii) a 
possible self-annihilation in space. In the following I will present a brief review of the requirement and state-of-the-art of these two types of simulations.

\subsection{Cosmological Simulations}

Data from wide-field galaxy surveys is used by cosmologists to perform mainly four "cosmic experiments" that constrain different aspects of $\Lambda$ CDM. 1) Using the clustering of galaxies and the apparent position of the BAO peak ${ }^{1}$ to measure the cosmic expansion history and the growth of structure 2) Measuring the relation between velocity and density fields via "redshift-space distortions" to probe the nature of gravity on cosmological scales. 3) Quantifying the abundance of galaxy clusters as a function of their mass to determine the probability distribution function (PDF) of density perturbations in the early Universe and the growth of structure. 4) Measuring the distortion in the apparent shape of high-redshift galaxies produced by weak gravitational lensing to directly measure the DM-dominated cosmic mass field.

Simulations of representative regions of our Universe provide the means to interpret these data, providing the expected hierarchy of correlation functions; the nonlinear density, velocity and potential fields; and the abundance and properties of dark matter halos. However, in order to provide accurate enough results, cosmic simulations need to match the volume of current and planned surveys, with high mass resolution to follow the formation of the dark matter structures expected to host observable galaxies. This is an extremely tough computational problem.

The first simulation to achieve these requirements was the Millennium-XXL [4]. It was carried in the summer of 2010 on the "JuRoPa" machine at the J'ulich Supercomputer Center (JSC) in Germany, employing 12888 computer cores and with an extremely memory efficient version of the Gadget- 3 code. The total computational time spent in the calculation was 2.8 million CPU hours. The MXXL followed more than 300 billion particles $\left(6720^{3}\right)$ representing the Dark Matter inside a box of $70 G p c^{3}$ volume and resolving large-scale structure with an unprecedented combination of volume and resolution. A realistic galaxy formation model was implemented, providing a sample of around 400 million galaxies at low redshift. The enormous statistical power of the simulation is hinted at in Fig 2, which shows the projected density field from the MXXL simulation together with four selected massive galaxy clusters.

Currently, the record-holder in terms of the number of simulation particles is the recently completed DEUS Full Universe Simulation [2], which uses 550 billion particles in a box of side length $21 h^{-1} \mathrm{Gpc}$. The calculation was performed using a version of the RAMSES code. It employed 10 million CPU hours and 38061 cores on the Curie supercomputer.

In terms of mass resolution for runs reaching $z=0$, the record-holder is the CosmoGrid simulation [10], which follows $2048^{3}$ particles, each of which of mass $1.28 \times 10^{5} M_{\odot}$, in a $30 h^{-1} \mathrm{Mpc}$ box. The calculation was carried on four different supercomputers in four different countries (Japan, United Kingdom, Finland and the Netherlands), which were employed concurrently for part of the run. The evolution was computed using the GreeM and SUSHI codes employing a total of 3.5 million CPU hours.

\footnotetext{
${ }^{1}$ The BAO are small-amplitude oscillatory features imprinted on the mass field of the Universe, which arise from sound waves in the baryon-radiation fluid prior to the epoch of recombination. The observed wavelength of these oscillations can be used as a "standard ruler" in the cosmos.
} 


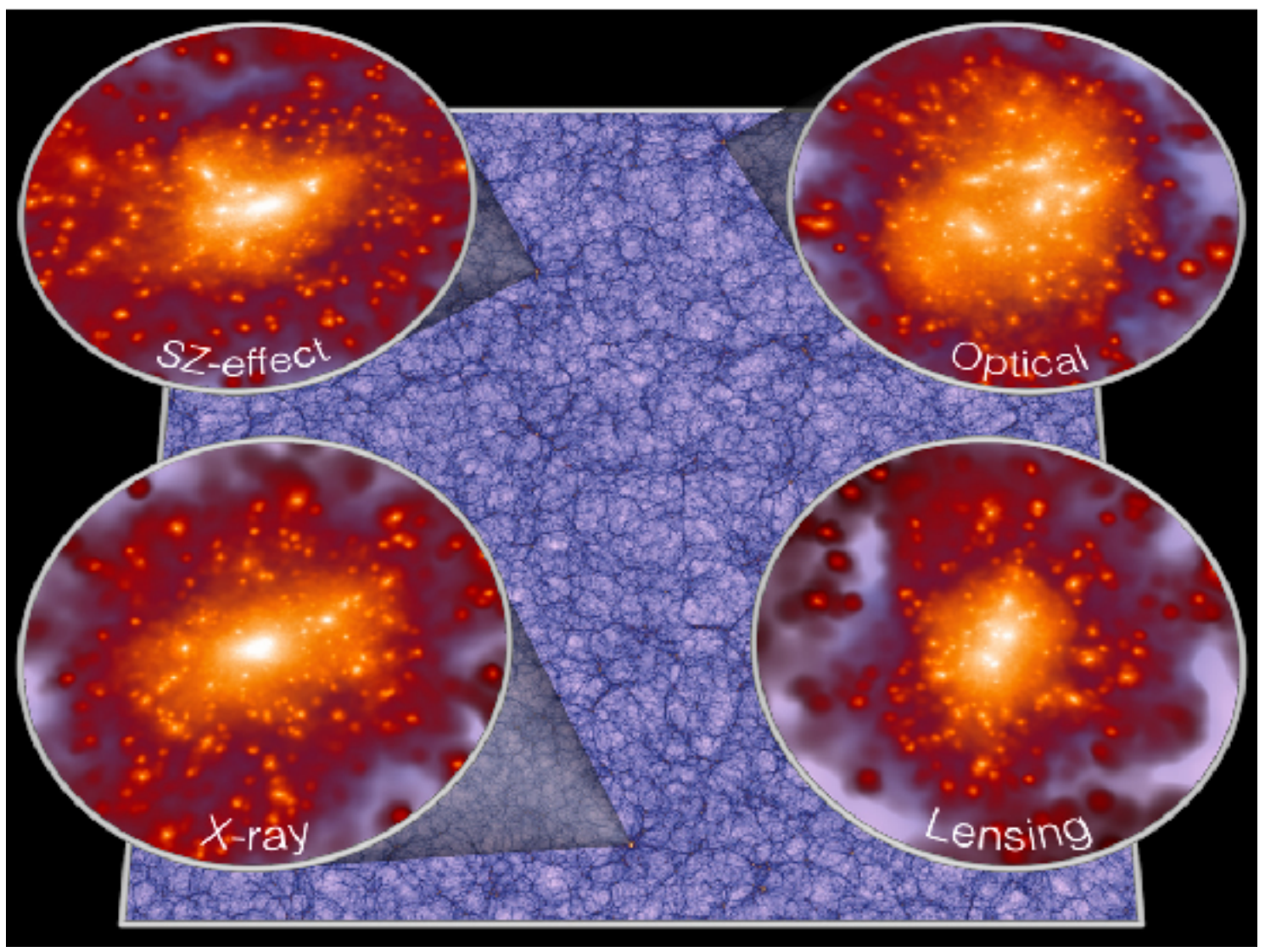

Figure 2: The Millennium-XXL simulation at redshift 0.25 on a slab $2050 \mathrm{Mpc}$ wide and $27 \mathrm{Mpc}$ deep. The insets correspond to the most extreme clusters identified according to different proxies for their observed properties: thermal Sunyaev-Zeldovich effect, Optical richness, X-ray emission and weak gravitational lensing signal. Adopted from [4]

\subsection{Zoom-in Simulations}

Complementing the statistical efforts described above, other type of simulations focuses all the computational resources in calculating the formation of individual halos. These numerical experiments have provided exquisite predictions for the distribution of the dark matter inside halos. They have revealed the existence of hundreds of thousands of gravitationally bound substructures, and resolved the distribution of dark matter with a resolution of the order of parsecs. In addition, they have characterized the internal structure of these systems; the abundance, properties and spatial distribution of subhalos and streams; the velocity structure; among other properties. This information has guided experimental searches for signatures of dark matter in detectors on earth as well as that of a possible self-annihilation of the dark matter particle.

The three most accurate calculations of this type are the Aquarius [21], Phoenix [8], and Via Lactea [13] projects, all of which resolve a single dark matter structure with over one billion particles The first of those projects simulated a large cluster-size DM halo, whereas the latter two focused on a Milky-Way size halo. Zoom-in simulations can typically achieve much higher mass 
resolution than full boxes. These runs have particle masses ranging from 100 to $10000 M_{\odot}$.

One should note that for a fixed number of particles, zoom-in simulations are much tougher computational problems than large-volume simulations for the codes and algorithms involved. This is because i) the much larger imbalances in the distribution of particles and in the force calculation, and ii) the wider range and larger number of timesteps required to simulate regions with very high densities and short dynamical times. Due to this, the above projects employed a similar amount of CPU time (2 to 4 million CPU hours each) as state-of-the-art full-box simulations, despite the latter following approximately two orders of magnitude more particles.

\section{Different Dark Matter flavours}

While all the major projects described in the previous section have assumed a standard $\Lambda \mathrm{CDM}$ Universe, there is a significant amount of effort in the community to simulate, and understand the impact of, more exotic cosmological components. These include, primordial departures from Gaussian fluctuations, massive neutrinos, self-interacting dark matter, modifications from general relativity (e.g., so-called f(R), Galileon models), different dark energy models (e.g., quintessence, Ratra-Peebles), interactions between dark energy and dark matter, among others (e.g., $[16,7,15$, $18,22])$.

Among the simulations with departures from $\Lambda \mathrm{CDM}$, there has been growing interest in scenarios with alternative dark matter particles. In particular, those in which the dark matter is warm instead of perfectly cold. The main motivation for such models is a history of discrepancies between predictions of CDM simulations and observations on small scales. Two of the most notable examples are i) the abundance of satellite galaxies orbiting around the Milky Way, ii) the density profiles of very small galaxies, iii) the central densities of classical dwarfs (i.e. massive Milky-Way satellites) as inferred from stellar kinematic. The largely-unknown physical processes involved in galaxy formation are usually advocated to reduce the tension. However, warm dark matter (WDM), is also an alternative to solve the aforementioned problems.

The topology of the Universe on large scales is identical between CDM and WDM (and so for many other variations of the DM model). However, the characteristics on small scales are very different. This is apparent in Fig 3, which shows the internal structure of a large dark matter halo simulated using cold (left panel) and warm dark matter (right panel). Because the properties of the DM particle appear on small highly nonlinear scales, accurate numerical simulations of WDM cosmologies are needed to observationally distinguish different candidates. This is not as simple as one might have naively expected.

It is now obvious to the community that WDM simulations have always been plagued by nonphysical numerical artifacts [23]. These effects appear as an artificial fragmentation of filaments into small and dense halos. These are extremely hard to avoid and outshine the real properties of the nonlinear density field. Therefore, there is reasonable doubt on the robustness and accuracy of the predictions of structure formation in WDM from N-body simulations, which could be due to the fundamental assumptions of the cosmological simulations and/or the discretization errors. These issues could also affect CDM simulations, although the magnitude of the problem is not clear.

In a recent paper, [3] showed that the new scheme described in 2.2 dramatically reduces the degree of artificial fragmentation in WDM. Thanks to this, it was possible to study in detail how the 

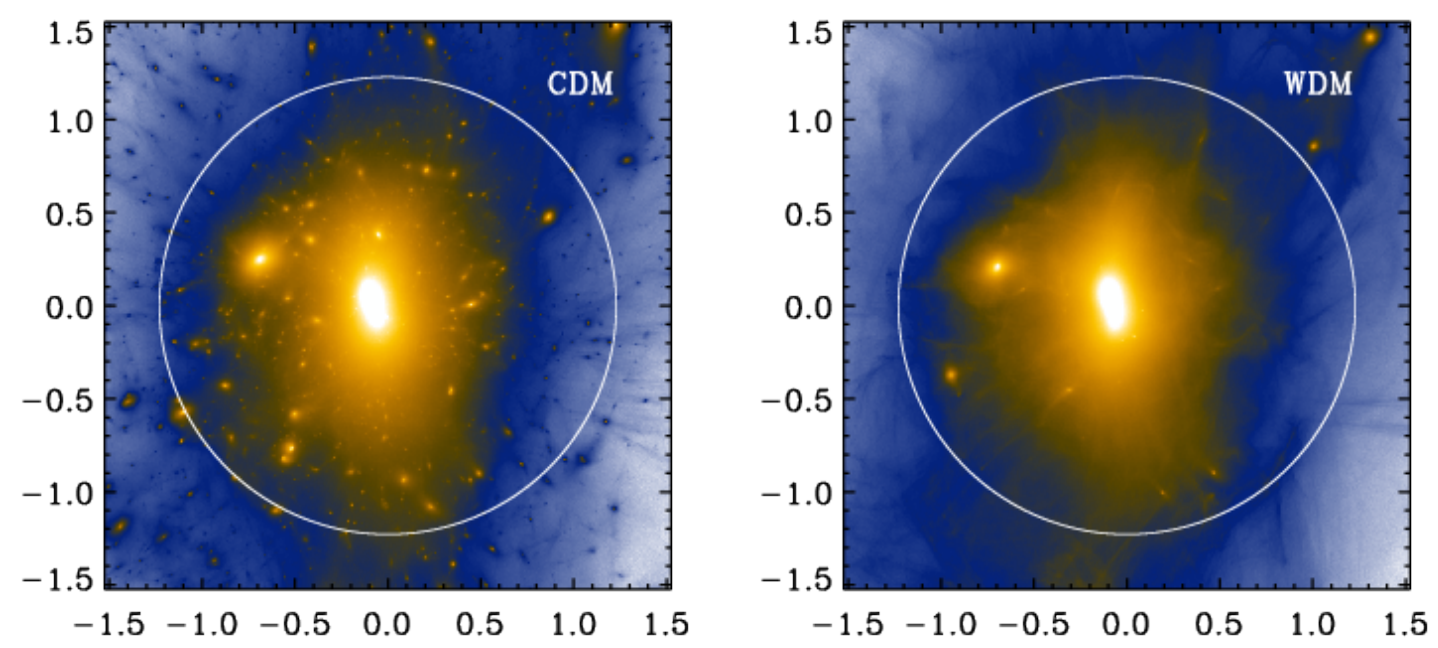

Figure 3: Projected dark matter density for a halo of mass $4.4 \times 10^{14} h^{-1} M_{\odot}$, simulated assuming CDM and a $250 \mathrm{eV}$ DM particle mass (left and right panels, respectively). The white circle indicates the virial radius, $1.2 h^{-1} \mathrm{Mpc}$ of the halos. Adopted from [3]

suppression of small-scale perturbations expected in WDM translates into a lack of low mass halos, and also to follow the collapse of the smallest halos in WDM cosmologies. These are promising results that show the room for potential improvements to current dark cosmological simulations.

\section{Summary and Outlook}

Dark cosmological simulations have a prime role in modern cosmology. These calculations follow the formation of structure of the Universe over a huge large dynamic range, from cosmic fields on Gigaparsecs scales down to the inner structure of the dark matter hosts of the smallest galaxies. Their results have helped establishing the standard model in cosmology, and nowadays they are guiding the design and helping in the interpretation of the latest generation of galaxy surveys and dark matter searches.

There are several areas in which one can foresee development in the next years. Firstly, dark cosmic simulations will continue the current trends towards larger volumes and higher resolution to more accurately predict structure formation in the Universe. In addition, the exploration of departures from $\Lambda \mathrm{CDM}$ will continue and most likely simulations will help to distinguish viable from ruled out models.

Similarly, zoom-In simulation will continue to increase mass resolution and the number of target objects, characterizing the hierarchy of dark matter structure more accurately. However, it is likely that the hydrodynamic effects will need to be included to achieve a faithful representation of the smallest scales.

Numerical algorithms are also likely to evolve in the near future. On one hand, this will be driven by higher accuracy. The recently developed methods following the distortions of phase- 
space volume elements offer an interesting path. On the other hand, computational efficiency and speed will require algorithms to adapt to heterogeneous and new computer architectures.

Further ahead in the future, the combination of high-resolution simulations with approximated methods could very quickly provide predictions for a grid of cosmological models (e.g., [5]). This would allow a direct employment of simulations to estimate cosmological parameters from data. In this way, a large range of observables and scales will be employed to learn more about the $\Lambda$ CDM model, exhaustively exploiting the huge amount of experimental data arriving over the next decade.

\section{Acknowledgements}

I would like to thank Tom Abel, Carlton Baugh, Carlos Frenk, Oliver Hahn, Adrian Jenkins, Ralf Kaehler, Mike Kuhlen, Volker Springel, Mark Vogelsberger and Simon White for countless discussions over the last few years, which have helped to shape the views and ideas presented here. I would also like to thank Oliver Hahn for comments on this manuscript, and to the organizers and participants of the "Frank N. Bash Symposium 2013" for an extremely enjoyable and stimulating meeting.

\section{References}

[1] T. Abel, O. Hahn, and R. Kaehler. Tracing the dark matter sheet in phase space. Monthly Notices of the RAS, 427:61-76, November 2012.

[2] J.-M. Alimi, V. Bouillot, Y. Rasera, V. Reverdy, P.-S. Corasaniti, I. Balmes, S. Requena, X. Delaruelle, and J.-N. Richet. DEUS Full Observable $\{\backslash$ Lambda $\}$ CDM Universe Simulation: the numerical challenge. ArXiv e-prints, June 2012.

[3] R. E. Angulo, O. Hahn, and T. Abel. How closely do baryons follow dark matter on large scales? ArXiv e-prints, January 2013.

[4] R. E. Angulo, V. Springel, S. D. M. White, A. Jenkins, C. M. Baugh, and C. S. Frenk. Scaling relations for galaxy clusters in the Millennium-XXL simulation. Monthly Notices of the RAS, 426:2046-2062, November 2012.

[5] R. E. Angulo and S. D. M. White. One simulation to fit them all - changing the background parameters of a cosmological N-body simulation. Monthly Notices of the RAS, 405:143-154, June 2010.

[6] G. Efstathiou, W. J. Sutherland, and S. J. Maddox. The cosmological constant and cold dark matter. Nature, 348:705-707, December 1990.

[7] F. Fontanot, V. Springel, R. E. Angulo, and B. Henriques. Semi-analytic galaxy formation in early dark energy cosmologies. Monthly Notices of the RAS, 426:2335-2341, November 2012.

[8] L. Gao, J. F. Navarro, C. S. Frenk, A. Jenkins, V. Springel, and S. D. M. White. The Phoenix Project: the dark side of rich Galaxy clusters. Monthly Notices of the RAS, 425:2169-2186, September 2012.

[9] O. Hahn, T. Abel, and R. Kaehler. A new approach to simulating collisionless dark matter fluids. Monthly Notices of the RAS, July 2013.

[10] T. Ishiyama, S. Rieder, J. Makino, S. Portegies Zwart, D. Groen, K. Nitadori, C. de Laat, S. McMillan, K. Hiraki, and S. Harfst. The Cosmogrid Simulation: Statistical Properties of Small Dark Matter Halos. The Astrophysical Journal, 767:146, April 2013. 
[11] R. Kaehler, O. Hahn, and T. Abel. A Novel Approach to Visualizing Dark Matter Simulations. arXiv:1208.3206, August 2012.

[12] M. Kilbinger, L. Fu, C. Heymans, F. Simpson, J. Benjamin, T. Erben, J. Harnois-Déraps, H. Hoekstra, H. Hildebrandt, T. D. Kitching, Y. Mellier, L. Miller, L. Van Waerbeke, K. Benabed, C. Bonnett, J. Coupon, M. J. Hudson, K. Kuijken, B. Rowe, T. Schrabback, E. Semboloni, S. Vafaei, and M. Velander. CFHTLenS: combined probe cosmological model comparison using 2D weak gravitational lensing. Monthly Notices of the RAS, 430:2200-2220, April 2013.

[13] M. Kuhlen, J. Diemand, P. Madau, and M. Zemp. The Via Lactea INCITE simulation: galactic dark matter substructure at high resolution. Journal of Physics Conference Series, 125(1):012008, July 2008.

[14] M. Kuhlen, M. Vogelsberger, and R. Angulo. Numerical simulations of the dark universe: State of the art and the next decade. Physics of the Dark Universe, 1:50-93, November 2012.

[15] B. Li, A. Barreira, C. M. Baugh, W. A. Hellwing, K. Koyama, S. Pascoli, and G.-B. Zhao. Simulating the quartic Galileon gravity model on adaptively refined meshes. Journal of Cosmology and Astroparticle Physics, 11:12, November 2013.

[16] A. Pillepich, C. Porciani, and O. Hahn. Halo mass function and scale-dependent bias from N-body simulations with non-Gaussian initial conditions. Monthly Notices of the RAS, 402:191-206, February 2010.

[17] Planck Collaboration, P. A. R. Ade, N. Aghanim, C. Armitage-Caplan, M. Arnaud, M. Ashdown, F. Atrio-Barandela, J. Aumont, C. Baccigalupi, A. J. Banday, and et al. Planck 2013 results. XVI. Cosmological parameters. ArXiv e-prints, March 2013.

[18] E. Puchwein, M. Baldi, and V. Springel. Modified-Gravity-GADGET: a new code for cosmological hydrodynamical simulations of modified gravity models. Monthly Notices of the RAS, 436:348-360, November 2013.

[19] A. G. Sánchez, C. G. Scóccola, A. J. Ross, W. Percival, M. Manera, F. Montesano, X. Mazzalay, A. J. Cuesta, D. J. Eisenstein, E. Kazin, C. K. McBride, K. Mehta, A. D. Montero-Dorta, N. Padmanabhan, F. Prada, J. A. Rubiño-Martín, R. Tojeiro, X. Xu, M. V. Magaña, E. Aubourg, N. A. Bahcall, S. Bailey, D. Bizyaev, A. S. Bolton, H. Brewington, J. Brinkmann, J. R. Brownstein, J. R. Gott, J. C. Hamilton, S. Ho, K. Honscheid, A. Labatie, E. Malanushenko, V. Malanushenko, C. Maraston, D. Muna, R. C. Nichol, D. Oravetz, K. Pan, N. P. Ross, N. A. Roe, B. A. Reid, D. J. Schlegel, A. Shelden, D. P. Schneider, A. Simmons, R. Skibba, S. Snedden, D. Thomas, J. Tinker, D. A. Wake, B. A. Weaver, D. H. Weinberg, M. White, I. Zehavi, and G. Zhao. The clustering of galaxies in the SDSS-III Baryon Oscillation Spectroscopic Survey: cosmological implications of the large-scale two-point correlation function. Monthly Notices of the RAS, 425:415-437, September 2012.

[20] S. Shandarin, S. Habib, and K. Heitmann. Cosmic web, multistream flows, and tessellations. Physical Review D, 85(8):083005, April 2012.

[21] V. Springel, J. Wang, M. Vogelsberger, A. Ludlow, A. Jenkins, A. Helmi, J. F. Navarro, C. S. Frenk, and S. D. M. White. The Aquarius Project: the subhaloes of galactic haloes. Monthly Notices of the RAS, 391:1685-1711, December 2008.

[22] F. Villaescusa-Navarro, F. Marulli, M. Viel, E. Branchini, E. Castorina, E. Sefusatti, and S. Saito. Cosmology with massive neutrinos I: towards a realistic modeling of the relation between matter, haloes and galaxies. ArXiv e-prints, November 2013.

[23] J. Wang and S. D. M. White. Discreteness effects in simulations of hot/warm dark matter. Monthly Notices of the RAS, 380:93-103, September 2007. 